\title{
External-field-induced Assembly for Biological Analytical Chemistry
}

\author{
Shiho TOKONAMI*,** \\ *Department of Applied Chemistry, Graduate School of Engineering, Osaka Prefecture University, 1-2, \\ Gakuen-cho, Naka, Sakai, Osaka 599-8570, Japan \\ **Research Institute for Light-induced Acceleration System (RILACS), Osaka Prefecture University, 1-2, \\ Gakuen-cho, Naka, Sakai, Osaka 599-8570, Japan
}

Guiding and concentrating analytes toward the observation and measurement sites by an external field such as light provide a key technology for non-contact, high-sensitivity, rapid, simple, and high-throughput bioanalysis.

Dr. Ashkin was awarded the Nobel Prize in Physics three years ago for the recognition of the optical tweezers that was induced by the focused laser with a high numerical aperture lens and applying them for biological systems. The technique has attracted many researchers in various research fields including analytical biochemistry especially for the application of highlyprecise manipulation of single living cells. ${ }^{1}$ In the 1990s, Masuhara et al. in Japan first applied the optical trap technique to the chemistry field. ${ }^{2}$ Their theoretical and experimental achievement of the separation analyses of nanomaterials such as metallic nanoparticles, carbon nanotubes, ${ }^{4}$ and nanodiamonds ${ }^{5}$ based on the quantum mechanical resonance effect in optical pressure $^{6}$ excited researchers in the field of physical chemistry.

Optical tweezers have widely been involved with various spectroscopies used in analytical chemistry. For example, Raman scattering spectroscopy ${ }^{7-11}$ is often suitable for the trapped analytes. In the presence of temperature-responsive poly( $N$-isopropylacrylamide), rhodamine $\mathrm{B}$ with a nM-level concentration was readily detected by the fluorescence and Raman scattering from the target molecules under the strong gradient force and photothermal effect of the plasmonic optical tweezers. $^{12}$ The temperature rise of a water droplet in the atmosphere levitated with the laser was measured by Raman spectroscopy to investigate characteristics of aerosols. ${ }^{13}$ The position of a microparticle with photochromic molecules levitated

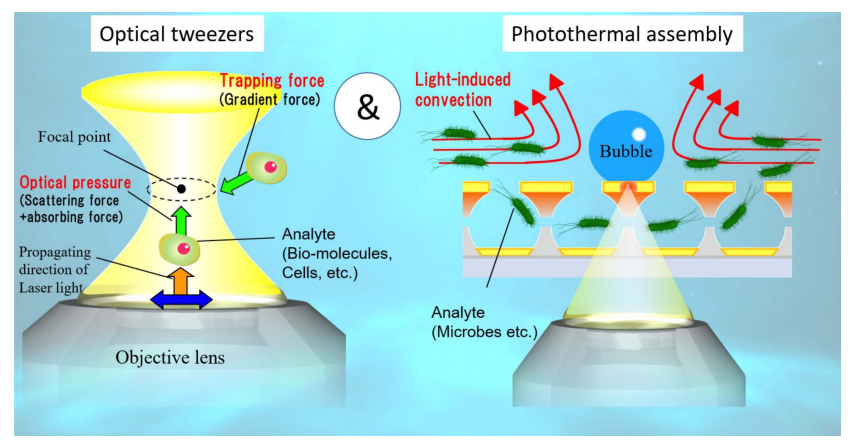

Analysis based on the trapping and condensation of biological samples by optical tweezers and photothermal assembly.

E-mail: tokonami@chem.osakafu-u.ac.jp in liquid was measured by the optical pressure of visible laser. ${ }^{14}$ In this case, the transfer of light momentum was resonantly enhanced by the appearance of a visible absorption peak according to photoisomerization by irradiating the ultraviolet light.

The control and analysis of the states of a matter using the optical trapping were also reported. Time required for the coalescence of oil droplets in an oil-in-water type emulsion by heating under the optical trapping was correlated with temperature in the Arrhenius plot. ${ }^{15}$ Thus, the use of the correlation is usefully applied to analyze materials in droplets. The suppression processes of Brownian motion and the crystallization occurred by prenucleation clusters of molecules were also studied under the optical trap. ${ }^{16}$ In medical applications, the solid-liquid phase transition of lipid bilayer membranes by heat occurred by Pt nanoparticles trapped with optical tweezers was applied to destroy the cultured cancer cells. ${ }^{17}$ Optical tweezers were also used to analyze the motion and mechanical properties of intracellular organelles and single molecules. ${ }^{18}$ For example, the generation of foliate pseudopodia at multiple sites in the cell and the effect on cell membrane tension by amphipathic peptides derived from the influenza M2 protein were evaluated. ${ }^{19}$

As shown in the examples above, the optical trapping is an excellent technique for highly precise manipulation of analytes. However, the range of action is limited only near the beam waist of the focused laser. On the other hand, it was suggested that the phtothermal effect cannot be ignored to concentrate analytes at the measurement site. ${ }^{20}$ The light-induced convection in photothermal assembly could contribute to accumulation of the analytes with micro- and nano-meter scales in a wide range of surroundings of the focused laser. For example, there is a report about micro-patterning of nanoparticles in the micron-meter order region using an assembly in photothermal convection near a light-induced bubble. ${ }^{21}$ The photo-assisted solvothermal method using plasmonic nanoparticles was studied to form indium hydroxide microcrystals in the region of about $10 \mu \mathrm{m} .^{22}$ In the field of bioanalysis, a rapid counting method by optical condensation of bacteria over a macroscopic region around a sub-millimeter-sized bubble generated on a flat gold nanoscale thin film was proposed. ${ }^{23}$ The effect of dissolved air was investigated via the relationship between the light-induced bubble and the Marangoni convection in degassed water. ${ }^{24}$

However, the heating effect inhibits the biorecognition events especially for the macromolecules, and it also damages the membrane of the living cells. For example, in order to solve this problem, the DNA detection based on the hybridization was softly accelerated by using both the optical force and 
photothermal convection, where the temperature was controlled below the melting temperature of double-stranded DNA. ${ }^{25}$ More recently, microbes such as bacteria have been trapped on the honeycomb-shaped substrates at a high density of $10^{6}-$ $10^{7}$ cells $/ \mathrm{cm}^{2}$ with a high survival rate of $80-90 \% .^{26}$ This is due to the suppression of heat transfer to the multi-pore structure as trapping sites. In addition, the function of electricityproducing bacteria is significantly increased by high condensation. As shown in these examples, the external fieldinduced assembly is a technology that is a promising tool in bioanalytical chemistry for accelerating specific detection and low-damage condensation. The integration of the theoretical and experimental approaches for controlling the photothermal effects is desired for the further development.

In this highlight, as an example of the analytical techniques using the external-field-induced assembly, the author focuses on the optical trap based on the electromagnetic effect of light and on the photothermal assembly using the thermohydrodynamic effect. By appropriate combination of the characteristics of both effects and skillful control of the photothermal effects of the probe particles and the substrate, non-contact and highly efficient condensation will be available at the measurement site avoiding damage on biological samples. Further research on optical condensation utilizing synergistic effect of optical force and photothermal effect will provide a new paradigm shift in analytical chemistry; for example, the detection of rare biological samples at a low-concentration that cannot be realized by the conventional methods, and the evaluation of metabolic function of a variety of microbes.

Keywords Optical tweezers, photothermal assembly, bioanalysis

\section{References}

1. A. Ashkin, J. M. Dziedzic, and T. Yamane, Nature, 1987, 330, 769 .

2. H. Masuhara, F. C. De Schryver, N. Kitamura, and N. Tamai (ed.), "Microchemistry: Spectroscopy and Chemistry in Small Domains", 1994, North-Holland, Amsterdam.

3. S. Ito, H. Yamauchi, M. Tamura, S. Hidaka, H. Hattori, T. Hamada, K. Nishida, S. Tokonami, T. Itoh, H. Miyasaka, and T. Iida, Sci. Rep., 2013, 3, 3047.

4. S. E. S. Spesyvtseva, S. Shoji, and S. Kawata, Phys. Rev.
Appl., 2015, 3, 044003.

5. H. Fujiwara, K. Yamauchi, T. Wada, H. Ishihara, and K. Sasaki, Sci. Adv., 2021, 7, eabd9551.

6. T. Iida and H. Ishihara, Phys. Rev. Lett., 2003, 90, 057403.

7. X. Li, L. Li, and Y. He, Anal. Sci., 2020, 36, 1025.

8. M. Horiguchi and S. Kunimura, Anal. Sci., 2020, 36, 1311.

9. W. Zhang, Z. Liu, Z. Liu, H. Qin, H. Li, H. Du, L. Fang, C. Wang, S. Zhang, and Z. Chen, Anal. Sci., 2020, 36, 1439.

10. M. J. Seo, L. Baek, and J. W. Ha, Anal. Sci., 2019, 35, 1009.

11. M. Banno, S. Takahashi, and H. Yui, Anal. Sci., 2019, 35, 911.

12. T. Shoji, D. Sugo, F. Nagasawa, K. Murakoshi, N. Kitamura, and Y. Tsuboi, Anal. Chem., 2017, 89, 532

13. S. Ishizaka, J. Ma, T. Fujiwara, K. Yamauchi, and N. Kitamura, Anal. Sci., 2016, 32, 425.

14. K. Setoura, A. M. Memon, S. Ito, Y. Inagaki, K. Mutoh, J. Abe, and H. Miyasaka, J. Phys. Chem. C, 2018, 122, 22033.

15. M. Mitsunobu, S. Kobayashi, N. Takeyasu, and T. Kaneta, Anal. Sci., 2017, 33, 709.

16. I. Hanasaki, K. Okano, H. Y. Yoshikawa, and T. Sugiyama, J. Phys. Chem. Lett., 2019, 10, 7452.

17. A. Samadi, H. Klingberg, L. Jauffred, A. Kjær, P. M. Bendix, and L. B. Oddershede, Nanoscale, 2018, 10, 9097.

18. K. Norregaard, R. Metzler, C. M. Ritter, K. Berg-Sørensen, and L. B. Oddershede, Chem. Rev., 2017, 117, 4342.

19. T. Masuda, K. Baba, T. Nomura, K. Tsujita, T. Murayama, T. Itoh, T. Takatani-Nakase, M. Sokabe, N. Inagaki, and S. Futaki, Commun. Biol., 2019, 2, 243.

20. A. Miyagawa and T. Okada, Anal. Sci., 2021, 37, 69.

21. S. Fujii, K. Kanaizuka, S. Toyabe, K. Kobayashi, E. Muneyuki, and M. Haga, Langmuir, 2011, 27, 8605.

22. H. M. L. Robert, F. Kundrat, E. Bermúdez-Ureña, H. Rigneault, S. Monneret, R. Quidant, J. Polleux, and G. Baffou, ACS Omega, 2016, $1,2$.

23. Y. Yamamoto, E. Shimizu, Y. Nishimura, T. Iida, and S. Tokonami, Opt. Mater. Express, 2016, 6, 1280.

24. K. Namura, S. Okai, S. Kumar, K. Nakajima, and M. Suzuki, Adv. Mater. Interfaces, 2020, 7, 2000483.

25. T. Iida, Y. Nishimura, M. Tamura, K. Nishida, S. Ito, and S. Tokonami, Sci. Rep., 2016, 6, 37768.

26. S. Tokonami, S. Kurita, R. Yoshikawa, K. Sakurai, T. Suehiro, Y. Yamamoto, M. Tamura, O. Karthaus, and T. Iida, Sci. Adv., 2020, 6, eaaz5757. 